\title{
A Stein Conjecture for the Circle
}

\author{
Jonathan Bennett ${ }^{1} \quad$ Anthony Carbery ${ }^{2} \quad$ Fernando Soria ${ }^{3}$ \\ Ana Vargas ${ }^{4}$ *
}

May 30, 2006

${ }^{1}$ School of Mathematics, University of Birmingham, Edgbaston, Birmingham, B15 2TT, England.

e-mail: J.Bennett@bham.ac.uk

${ }^{2}$ School of Mathematics, University of Edinburgh, King's Buildings, Mayfield Road, Edinburgh, EH9 3JZ, Scotland.

e-mail: A.Carbery@ed.ac.uk

3 Departamento de Matemáticas, Universidad Autónoma de Madrid, 28049, Madrid, Spain.

e-mail: fernando.soria@uam.es

${ }^{4}$ Departamento de Matemáticas, Universidad Autónoma de Madrid, 28049, Madrid, Spain.

e-mail: ana.vargas@uam.es

\begin{abstract}
We discuss the manner in which one might expect directional maximal functions to control the Fourier extension operator via $L^{2}$ weighted inequalities. We prove a general inequality of this type for the extension operator restricted to circles in the plane.
\end{abstract}

\section{Introduction}

In the Proceedings of the 1978 Williamstown Conference on Harmonic Analysis, E. M. Stein [S] raised the question of the manner in which one might expect Kakeya or Nikodym type maximal functions to control the disc multiplier or Bochner-Riesz multiplier operators via $L^{2}$ weighted inequalities. For example, if $S$ represents the disc multiplier operator on $\mathbb{R}^{n}$,

$$
(S f)^{\wedge}(\xi)=\chi_{\{|\xi| \leq 1\}} \widehat{f}(\xi)
$$

*All authors were supported by EC projects "Harmonic Analysis" and "HARP"; the second also by a Leverhulme Fellowship, and the third and fourth by MCyT grant BFM2001-0189. 
and $\mathcal{M}$ is the universal maximal function on $\mathbb{R}^{n}$ obtained by taking maximal averages over arbitrary rectangles in $\mathbb{R}^{n}$, is there an inequality such as

$$
\int|S f(x)|^{2} w(x) d x \leq C_{s} \int|f(x)|^{2}\left[\mathcal{M} w^{s}(x)\right]^{\frac{1}{s}} d x
$$

(each $s>1$ )? (Stein considered the question in two dimensions, and it should be pointed out that this is only one of many possible formulations - not the one originally made by Stein.)

Very little progress has been made on this difficult problem. In [CRS], Romera and two of the present authors established that (1) does indeed hold if the weight $w$ is radial, and in fact implicit in that work was a result for Bochner-Riesz operators. Let, for $\delta$ small,

$$
\left(S_{\delta} f\right)^{\wedge}(\xi)=\Phi\left(\frac{|\xi|-1}{\delta}\right) \widehat{f}(\xi)
$$

where $\Phi$ is a standard non-negative normalised bump function of one real variable. Then, for each $\delta$, and $w$ radial

$$
\int\left|S_{\delta} f(x)\right|^{2} w(x) d x \leq C \int|f(x)|^{2} \mathcal{M} w(x) d x
$$

It seems very likely that a more quantitative version of (2) holds, viz.

$$
\int\left|S_{\delta} f(x)\right|^{2} w(x) d x \leq C \int|f(x)|^{2} \mathcal{M}^{\delta} w(x) d x
$$

for $w$ radial, where $\mathcal{M}^{\delta}$ is the maximal operator obtained by taking maximal averages over all rectangles of eccentricity less than $\delta^{-1}$.

It has long been known that the restriction phenomenon for the Fourier transform is closely related to the disc and Bochner-Riesz multipliers. Let the dual to the restriction operator be

$$
g \longmapsto \widehat{g d \sigma}
$$

(the extension operator) where $g \in L^{1}\left(\mathbb{S}^{n-1}\right)$. This is a map from $L^{1}\left(\mathbb{S}^{n-1}\right)$ to $C\left(\mathbb{R}^{n}\right)$. In $[\mathrm{CSV}]$ it was shown that certain weighted inequalities for $S_{\delta}$ and the extension operator are equivalent:

Proposition 1 ([CSV]) Let $w$ be a weight supported in $\{|x| \leq 1\}$. The following statements are equivalent, with $\delta=\frac{1}{R}$ :

(i) There exists a constant A such that

$$
\int_{|x| \leq 1}\left|S^{\delta} f(x)\right|^{2} w(x) d x \leq A \int_{\mathbb{R}^{n}}|f(x)|^{2} d x
$$

for all $f \in L^{2}\left(\mathbb{R}^{n}\right)$, ( a certain $\left.0<\delta \leq 1\right)$. 
(ii) There exists a constant B such that

$$
\int_{|x| \leq 1}|\widehat{g d \sigma}(R x)|^{2} w(x) d x \leq \frac{B}{R^{n-1}}\|g\|_{L^{2}\left(\mathbb{S}^{n-1}\right)}^{2}
$$

for all $g \in L^{2}\left(\mathbb{S}^{n-1}\right)$, (a certain $\left.R \geq 1\right)$.

Moreover the constants $A$ and $B$ are equivalent.

Here, $S^{\delta}$ is a rescaled version of $S_{\delta}$ which operates at unit scale; that is, the Fourier multiplier of $S^{\delta}$ is $\Phi\left(|\xi|-\delta^{-1}\right)$ instead of $\Phi\left(\frac{|\xi|-1}{\delta}\right)$ for $S_{\delta}$.

Thus, were one to entertain the possibility that Stein's conjecture in the form (3) held for arbitrary weights $w$, an immediate consequence would be

$$
\int_{|x| \leq 1}|\widehat{g d \sigma}(R x)|^{2} w(x) d x \leq \frac{C}{R^{n-1}}\|g\|_{2}^{2} \sup \frac{w(T)}{|T|}
$$

where the sup is taken over all tubes $T$ in the unit ball of eccentricity less than $R$; one would then be tempted to consider the possibility of weighted inequalities such as

$$
\int_{|x| \leq 1}|\widehat{g d \sigma}(R x)|^{2} w(x) d x \leq \frac{C}{R^{n-1}} \int_{\mathbb{S}^{n-1}}|g(\tau)|^{2} \sup _{T \| \tau} \frac{w(T)}{|T|} d \sigma(\tau)
$$

(where the sup is taken over all tubes $T$ in the unit ball of eccentricity less than $R$, which are parallel to $\tau$ ). Of course (5) would be an immediate consequence of (6), and (6) represents an analogue of Stein's conjecture for the extension operator; we note that the operator

$$
w \longmapsto \sup _{T \| \tau} \frac{w(T)}{|T|}
$$

is a variant of the Kakeya (as opposed to Nikodym) maximal function, taking functions on $\mathbb{R}^{n}$ to functions on $\mathbb{S}^{n-1}$.

It should be pointed out that inequalities such as (4) and (5) have arisen in the work of Barceló, Ruiz and Vega [BRV] on weighted estimates for solutions to the Helmholtz equation, and in the work of Carbery and Soria ([CS1], [CS2]) and Carbery, Soria and Vargas [CSV] on localisation for the inverse Fourier transform in $\mathbb{R}^{n}$. Testing (4) on standard bump functions leads one to consider the validity of

$$
\int_{|x| \leq 1}|\widehat{g d \sigma}(R x)|^{2} w(x) d x \leq \frac{C}{R^{n-1}}\|g\|_{2}^{2} \sup _{\substack{T=T\left(\alpha, \alpha^{2} R\right) \\ R^{-1} \leq \alpha \leq R^{-1 / 2}}} \frac{w\left(T\left(\alpha, \alpha^{2} R\right)\right)}{\alpha^{n-1}},
$$

where $T\left(\alpha, \alpha^{2} R\right)$ denotes a tube in $\mathbb{R}^{n}$ of $n-1$ short sides of length $\alpha$ and one long side of length $\alpha^{2} R$. If true, this inequality would represent a strengthening of (5). 
The main subject of this work is inequality (6); that is, Stein's conjecture for the extension operator. For $w$ radial, the maximal operator on the right hand side of (6) is constant, and so (6) for radial $w$ is an immediate consequence of (5). In this paper we look at the "opposite" situation, where the weights are "trivial" in the radial direction but are arbitrary in the angular direction. Theorem 2, the main theorem of the paper, is a variant of (6) for weights - or densities - supported on $\mathbb{S}^{1}$, and represents the first progress on Stein's Conjecture since [CRS]. This work, which is the content of Section 2, also gives sharp weighted inequalities for certain highly oscillatory convolution operators on $\mathbb{S}^{1}$. For various reasons, our results are satisfactory only in dimension two.

Notation. For non-negative real numbers $A$ and $B$ we write $A \lesssim B(A \gtrsim B)$ if there exists an absolute constant $c>0$ such that $A \leq c B(A \geq c B)$. We write $A \sim B$ if both $A \lesssim B$ and $A \gtrsim B$.

We shall, in what follows, often identify $\theta \in[-\pi, \pi],(\cos \theta, \sin \theta) \in \mathbb{S}^{1} \subset \mathbb{R}^{2}$, and $e^{i \theta} \in \mathbb{T} \subset \mathbb{C}$, without further mention.

For notational simplicity, whenever $\mu$ is a density on $\mathbb{R}^{2}$ with $\operatorname{supp}(\mu) \subset$ $\mathbb{S}^{1}$, we also denote by $\mu$ the corresponding density on $\mathbb{S}^{1}$. The context of our arguments will remove any ambiguity in making this identification.

\section{Densities supported on $\mathbb{S}^{1}$.}

Our main result is the following.

Theorem 2 For all $R \geq 1$, and measures $\mu$ supported on $\mathbb{S}^{1}$,

$$
\int_{\mathbb{S}^{1}}|\widehat{g d \sigma}(R x)|^{2} d \mu(x) \lesssim \frac{\log R}{R} \int_{\mathbb{S}^{1}}|g(\omega)|^{2} M \mathcal{M}^{R}(\mu)(\omega) d \sigma(\omega),
$$

and

$$
\int_{\mathbb{S}^{1}}|\widehat{g d \sigma}(R x)|^{2} d \mu(x) \lesssim \frac{1}{R} \int_{\mathbb{S}^{1}}|g(\omega)|^{2} M \mathcal{M}^{R} M^{2}(\mu)(\omega) d \sigma(\omega)
$$

where

$$
\mathcal{M}^{R} \mu(\omega)=\sup _{\substack{T \| \omega, R^{-1} \leq \alpha \leq R^{-2 / 3}}} \frac{\mu\left(T\left(\alpha, \alpha^{2} R\right)\right)}{\alpha},
$$

and $M$ is the Hardy-Littlewood maximal function on $\mathbb{S}^{1}$.

Here $T(\alpha, \beta)$ denotes a rectangle in the plane, of short side $\alpha$ and long side $\beta$.

The above theorem may be thought of as Stein-type inequalities for densities $\mu$ on the circle. The form of the maximal function $\mathcal{M}^{R}$ is strongly suggested by the standard examples in this context; i.e. $g(\omega)$ of the form $e^{i a \cdot \omega} \chi(\omega)$, where $\chi$ is the characteristic function of a cap on $\mathbb{S}^{1}$ and $a \in \mathbb{R}^{2}$. It should be pointed out that the maximal function $\mathcal{M}^{R}$ is closely related to the functional appearing in (8), and is in general much smaller than the one appearing in (6). The two inequalities (9) and (10) are of course very similar. In the second we avoid the 
logarithmic factor, at the expense of having a slightly larger maximal operator on the right hand side. However, the impact of the extra factors of $M$ in (10) is limited; in particular, it is easy to see that $\left\|\mathcal{M}^{R} M^{2} \mu\right\|_{\infty}$ and $\left\|\mathcal{M}^{R} \mu\right\|_{\infty}$ only differ by factors which are at worst $(\log R)^{2}$. The two corollaries that we now give illustrate some of the virtues of both (9) and (10).

An immediate consequence of (9) is the following analogue of inequality (8).

Corollary 3 For $g \in L^{2}\left(\mathbb{S}^{1}\right)$, $\mu$ a measure supported on $\mathbb{S}^{1}$ and $R \geq 1$,

$$
\int_{\mathbb{S}^{1}}|\widehat{g d \sigma}(R x)|^{2} d \mu(x) \lesssim \frac{\log R}{R}\|g\|_{2}^{2} \sup _{R^{-1} \leq \alpha \leq R^{-2 / 3}} \frac{\mu\left(T\left(\alpha, \alpha^{2} R\right)\right)}{\alpha} .
$$

Inequalities (9) and (10) are sharp in a number of ways. In particular, the optimal $L^{p}$ inequalities for $\mathcal{M}^{R}$ imply those for the extension operator (when restricted to circles) by a direct application of Hölder's inequality in (10). This is the source of our next corollary, whose proof we leave until the end of the section.

\section{Corollary 4}

$$
\|\widehat{g d \sigma}(R \cdot)\|_{L^{3}\left(\mathbb{S}^{1}\right)} \lesssim R^{-1 / 3}\|g\|_{L^{3}\left(\mathbb{S}^{1}\right)}
$$

for all $g \in L^{3}\left(\mathbb{S}^{1}\right)$ and $R \geq 1$.

We remark that (11) is a known inequality, and can be seen as a consequence of work of Greenleaf and Seeger [GS].

\section{Remarks.}

1. It is important to point out that the operator

$$
\left.g \longmapsto \widehat{g d \sigma}(R \cdot)\right|_{\mathbb{S} 1}
$$

coincides with convolution on $\mathbb{S}^{1}$ with $e^{i R \cos }$. Thus Theorem 2 may be viewed as two-weighted inequalities for convolution with $e^{i R \cos }$. For example, inequality (10) is equivalent to

$$
\int_{\mathbb{S}^{1}}\left|e^{i R \cos *} * g\right|^{2} d \mu \lesssim \frac{1}{R} \int_{\mathbb{S}^{1}}|g|^{2} M \mathcal{M}^{R} M^{2}(\mu) d \sigma,
$$

for all measures $\mu$ on the circle and $R \geq 1$. Naturally, Corollaries 3 and 4 may be interpreted in a similar way.

2. It is not difficult to show that if $S$ is any $\rho \times \rho^{2} R$ tube, $R^{-1} \leq \rho \leq R^{-1 / 2}$ and $\mu$ is any measure supported on $\mathbb{S}^{1}$, then

$$
\frac{\mu\left(S\left(\rho, \rho^{2} R\right)\right)}{\rho} \lesssim \sup _{\substack{T \| S \\ R^{-1} \leq \alpha \leq R^{-2 / 3}}} \frac{\mu\left(T\left(\alpha, \alpha^{2} R\right)\right)}{\alpha} .
$$

Thus Theorem 2 and Corollary 3 are consistent with inequality (8) discussed in the introduction. 
3. The maximal function $\mathcal{M}^{R}$ appearing in Theorem 2 is smaller than $\widetilde{\mathcal{M}}^{R}$, in which the sup is taken over $R^{-1} \leq \alpha \leq R^{-1 / 2}$. This is due to reasons allied with Remark 2 immediately above. Note that if for a rectangle $T$, $\operatorname{ecc}(T)$ denotes its eccentricity, then

$$
\sup _{T \| \theta} \frac{\nu\left(T\left(R^{-1 / 2}, 1\right)\right)}{R^{-1 / 2}} \leq \widetilde{\mathcal{M}}^{R} \nu(\theta) \leq \sup _{\begin{array}{c}
T \| \theta \\
\operatorname{ecc}(T) \leq R^{1 / 2}
\end{array}} \frac{\nu(T)}{|T|}
$$

and

$$
\mathcal{M}^{R} \nu(\theta) \leq \frac{1}{R^{1 / 3}} \sup _{\substack{T \| \theta \\ \operatorname{ecc}(T) \leq R^{1 / 3}}} \frac{\nu(T)}{|T|}
$$

\section{A Brief Overview of the Proof.}

Inequalities (9) and (10) encode (in $\mathcal{M}^{R}$ ) geometric information about the action of $g \mapsto e^{i R \cos *} * g$ at every scale (one only needs to localise the support of $\mu$ at different scales to see this). In order to prove (9) and (10) we must somehow identify this "geometric action" on a general $g$ on $\mathbb{S}^{1}$. However, for a general $g$, it turns out that this action is encrypted in a non-trivial way. More specifically, convolution with $e^{i R \cos } \cdot$ acts on certain particular frequency blocks of $g$ in different ways. To see this geometric action one has to decompose $g$ depending on its frequencies at a range of scales, on each of which the "geometric action" is no longer subject to "interference". This action can then be seen to be essentially a rotation through $\pi / 2$ (modulo certain multiplying factors of Bessel functions). In this way such a decomposition is very much like an eigenfunction expansion. However, in general one pays a very heavy price for performing frequency decompositions, since they fail to exhibit any orthogonality on $L^{2}(d \mu),(d \mu$ being arbitrary). It is therefore crucial that the frequency decomposition that we choose is as "economical" as possible. The identification of this "optimal" frequency decomposition is therefore the key ingredient in our proof.

Let us describe briefly the main sequence of steps we take in our argument. We make two consecutive decompositions of the frequencies of $g$ (the first "dyadic", and the second "equally spaced"). The first is dictated by the asymptotic behaviour of the Bessel functions $\left\{J_{k}(R)\right\}$ (the Fourier multiplying sequence associated with convolution with $\left.e^{i R \cos \cdot}\right)$. We then fix a term in this initial decomposition, and observe that the restricted frequency support allows us to perform a certain scale of local averaging on the measure $\mu$ (or equivalently, to suppose that $\mu$ is essentially constant at a certain scale). This then leads us to claim that (9) and (10) hold (for such a piece) where the supremum in the maximal function $\mathcal{M}^{R}$ is restricted to a fixed scale of rectangle $T$. It is then pertinent to take "local suprema" of $\mu$ at a scale dictated by the eccentricity of the rectangles that we permit. This increased level of "constancy", or "smoothness", of $\mu$, in turn provokes the second frequency decomposition of $g$. It is on the pieces of this final decomposition where we observe the "geometric 
action" of convolution with $e^{i R \cos }$. Finally, we put together the pieces in an appropriate way using Littlewood-Paley arguments.

We will need the following adaptation of a well-known uniform Bessel function estimate; see for example Romera $[\mathrm{R}]$.

For each $\theta \in[-\pi, \pi], k \in \mathbb{Z}$ and $s \geq 1$, let

$$
J_{k}^{\theta}(s)=\int_{-\pi}^{\theta} e^{i(k t+s \cos t)} d t .
$$

Lemma 5 There exists an absolute constant $c$ such that

$$
\left|J_{k}^{\theta}(s)\right| \leq c s^{-1 / 2} \min \left\{k^{1 / 6},\left|\frac{|s|+|k|}{|s|-|k|}\right|^{1 / 4}\right\},
$$

for all $s, k$ and $\theta$.

Proof. The uniformity in $\theta$ requires little effort since Lemma 5 may be proved using routine applications of the classical Van der Corput Lemma - see [S1]. In particular, for $|k| \leq s / 2,|k| \geq 3 s / 2$ and $s-s^{1 / 3} \leq|k| \leq s+s^{1 / 3}$, the claimed bound follows immediately. We now deal with the two remaining cases. Suppose first that $s / 2 \leq|k| \leq s-s^{1 / 3}$. Let

$$
E=\left\{t \in[-\pi, \theta]:\left|h^{\prime}(t)\right| \lesssim\left(s^{2}-k^{2}\right)^{1 / 2}\right\},
$$

where $h(t)=k t+s \cos t$. By elementary trigonometry, $\left|h^{\prime \prime}(t)\right| \gtrsim\left(s^{2}-k^{2}\right)^{1 / 2}$ for all $t \in E$. Hence by Van der Corput's Lemma,

$$
\left|\int_{E} e^{i h(t)} d t\right| \lesssim\left(s^{2}-k^{2}\right)^{-1 / 4} \sim s^{-1 / 2}\left|\frac{|s|+|k|}{|s|-|k|}\right|^{1 / 4},
$$

and

$$
\left|\int_{[-\pi, \theta] \backslash E} e^{i h(t)} d t\right| \lesssim\left(s^{2}-k^{2}\right)^{-1 / 2},
$$

which is also bounded by the required quantity uniformly in $\theta$. The remaining case $s+s^{1 / 3} \leq|k| \leq 3 s / 2$ may be handled in a similar standard way.

\subsection{The Proof of Theorem 2.}

The proof we give consists principally in establishing one main proposition, which we now formulate. How this proposition implies Theorem 2 will be dealt with afterwards.

Fix a $p$ with $1 \leq 2^{p} \leq R^{2 / 3}$; we shall decompose $g$ according to its Fourier frequencies in a certain manner. (We tacitly assume that $R^{2 / 3}$ is a power of 2 in what follows merely for notational convenience.) Let

$$
\mathcal{A}_{p}= \begin{cases}\left\{j \in \mathbb{Z}: R-j \sim 2^{-p} R\right\}, & \text { if } 1<2^{p}<R^{2 / 3} \\ \left\{j \in \mathbb{Z}: 0 \leq R-j \leq R^{1 / 3}\right\}, & \text { if } 2^{p}=R^{2 / 3}\end{cases}
$$




$$
\begin{aligned}
& \mathcal{B}_{p}= \begin{cases}\left\{j \in \mathbb{Z}: j-R \sim 2^{-p} R\right\}, & \text { if } 1<2^{p}<R^{2 / 3} \\
\left\{j \in \mathbb{Z}: 0 \leq j-R \leq R^{1 / 3}\right\}, & \text { if } 2^{p}=R^{2 / 3},\end{cases} \\
& \mathcal{C}_{0}=\{j \in \mathbb{Z}:|j| \leq R / 2\},
\end{aligned}
$$

and

$$
\mathcal{C}_{\infty}=\{j \in \mathbb{Z}:|j|>3 R / 2\} .
$$

Thus the $\mathcal{A}_{p}$ 's together with the $-\mathcal{A}_{p}$ 's, $\mathcal{B}_{p}^{\prime} s,-\mathcal{B}_{p}^{\prime} s, \mathcal{C}_{0}$ and $\mathcal{C}_{\infty}$ form an (approximate) partition of $\mathbb{Z}$.

For each integer $p$ with $1 \leq 2^{p} \leq R^{2 / 3}$ we define a certain maximal function $\mathcal{M}_{p}$ as follows. If $\nu$ is a measure supported on the unit ball $\mathbb{B} \subset \mathbb{R}^{2}, \mathcal{M}_{p}(\nu)$ is defined for $\omega \in \mathbb{S}^{1}$ by

$$
\mathcal{M}_{p} \nu(\omega)=\sup _{T \| \omega} \frac{\nu\left(T\left(2^{p / 2} / R, 2^{p} / R\right)\right)}{2^{p / 2} / R} .
$$

Proposition 6 Let $\mu$ be a measure supported on $\mathbb{S}^{1}$.

1. If $g$ has Fourier frequencies supported in either $\mathcal{A}_{p},-\mathcal{A}_{p}, \mathcal{B}_{p}$ or $-\mathcal{B}_{p}$, then

$$
\int_{\mathbb{S}^{1}}|\widehat{g d \sigma}(R x)|^{2} d \mu(x) \lesssim \frac{1}{R} \int_{\mathbb{S}^{1}}|g(\omega)|^{2} \mathcal{M}_{p}(\mu)(\omega) d \sigma(\omega) .
$$

2. If $g$ has Fourier frequencies supported in either $\mathcal{C}_{0}$ or $\mathcal{C}_{\infty}$, then

$$
\int_{\mathbb{S}^{1}}|\widehat{g d \sigma}(R x)|^{2} d \mu(x) \lesssim \frac{1}{R} \int_{\mathbb{S}^{1}}|g(\omega)|^{2} \mathcal{M}_{0}(\mu)(\omega) d \sigma(\omega) .
$$

\section{Proof of Proposition 6.}

We begin with part 1. Let us suppose that $g$ has Fourier frequencies supported in $\mathcal{A}_{p}$. The analysis required in order to treat the remaining cases involves only trivial modifications.

By the standard formula for the Fourier transform of the density $e^{i j \theta} d \sigma$, if

$$
g(\theta)=\sum_{j \in \mathcal{A}_{p}} \alpha_{j} e^{i j \theta}
$$

then for $|x|=1$,

$$
\widehat{g d \sigma}(R x)=\sum_{j \in \mathcal{A}_{p}} \alpha_{j} J_{j}(R) e^{i j \arg (x)},
$$

where $J_{j}$ denotes the Bessel function of order $j$. 
Now,

$$
\begin{aligned}
\int_{\mathbb{S}^{1}}|\widehat{g d \sigma}(R x)|^{2} d \mu(x) & =\sum_{j, k \in \mathcal{A}_{p}} \alpha_{j} \bar{\alpha}_{k} J_{j}(R) J_{k}(R) \int_{-\pi}^{\pi} e^{i(j-k) \theta} d \mu \\
& =\sum_{j, k \in \mathcal{A}_{p}} \alpha_{j} \bar{\alpha}_{k} J_{j}(R) J_{k}(R) \widehat{\mu}(k-j) \\
& =\sum_{j, k \in \mathcal{A}_{p}} \alpha_{j} \bar{\alpha}_{k} J_{j}(R) J_{k}(R) \widehat{P}_{p}(k-j) \widehat{\mu}(k-j) \\
& =\int_{\mathbb{S}^{1}}|\widehat{g d \sigma}(R x)|^{2} P_{p} * \mu(x) d \sigma
\end{aligned}
$$

for any $P_{p}$ satisfying $\widehat{P_{p}}(j) \equiv 1$ whenever $|j| \leq 4.2^{-p} R$. We can clearly choose $P_{p}$ to be the kernel of an approximation to the identity on $\mathbb{S}^{1}$ at scale $2^{p} / R$ such that for each $N \in \mathbb{N}$,

$$
\left|P_{p}(\phi)\right| \lesssim \frac{2^{-p} R}{\left(1+2^{-p} R|\phi|\right)^{N}}
$$

for all $\phi \in[-\pi, \pi]$. (For our purposes $N=2$ will suffice here.)

The form of the weight we aim to have on the right hand side of the inequality suggests we dominate $P_{p} * \mu$ pointwise by

$$
\psi_{1}(\theta)=\sup _{|\eta-\theta| \leq 2^{-p / 2}}\left|P_{p} * \mu(\eta)\right|
$$

(a smaller scale of local supremum here leads to the failure of Lemma 9 below for general $p$ ). We will exploit this increased level of constancy on the frequency side as follows. Let $\Theta_{p}$ be a non-negative function on $\mathbb{S}^{1}$ with non-negative Fourier coefficients supported in $\left\{j \in \mathbb{Z}:|j| \leq 2^{p / 2}\right\}$. We may choose $\Theta_{p}$ so that

(i) for each $N \in \mathbb{N}$,

$$
\Theta_{p}(\phi) \lesssim \frac{2^{p / 2}}{\left(1+2^{p / 2}|\phi|\right)^{N}}, \quad \text { and }
$$

(ii) there is an absolute constant $c>0$ (independent of $p$ ) for which $\Theta_{p}(\phi) \gtrsim$ $2^{p / 2}$ whenever $|\phi| \leq c 2^{-p / 2}$.

(For our purposes (15) with $N=3$ will suffice.) We now let $\psi_{2}=\Theta_{p} * \psi_{1}$.

Lemma $7 \psi_{1} \lesssim \psi_{2}$.

Proof. By the properties of $\Theta_{p}$,

$$
\Theta_{p} * \psi_{1}(\theta) \gtrsim 2^{p / 2} \int_{|\phi| \leq c 2^{-p / 2}} \psi_{1}(\theta-\phi) d \phi .
$$

By elementary considerations, either

$$
\psi_{1}\left(\theta^{\prime}\right) \geq \psi_{1}(\theta) \text { for all } \theta-2^{-p / 2} \leq \theta^{\prime} \leq \theta,
$$


Or

$$
\psi_{1}\left(\theta^{\prime}\right) \geq \psi_{1}(\theta) \text { for all } \theta \leq \theta^{\prime} \leq \theta+2^{-p / 2},
$$

and so $\Theta_{p} * \psi_{1}(\theta) \gtrsim \psi_{1}(\theta)$ uniformly in $\theta$.

By the above lemma it suffices to control

$$
\int_{\mathbb{S}^{1}}|\widehat{g d \sigma}(R x)|^{2} \psi_{2}(x) d \sigma(x) .
$$

The fact that the frequencies of $\psi_{2}$ are supported in $\left\{|j| \leq 2^{p / 2}\right\}$ leads us to make a further frequency decomposition as follows. For $L \in \mathbb{N}$, let $W_{L}$ be a function on $\mathbb{S}^{1}$ with frequencies supported in $\{j \in \mathbb{Z}:|j| \leq 2 L\}$ such that

$$
\sum_{k} \widehat{W}_{L}(j+k L)=1
$$

for all $j \in \mathbb{Z}$. In addition, let us choose (as we may) $W_{L}$ such that for each $N \in \mathbb{N}$

$$
\left|W_{L}(\phi)\right| \lesssim \frac{L}{(1+L|\phi|)^{N}}
$$

for all $\phi \in[-\pi, \pi]$. (Again, for our purposes, (17) with $N=3$ will suffice here.)

If for each $0 \leq q \leq 2^{-3 p / 2} R$ we write

$$
g_{q}(\theta)=\int_{-\pi}^{\pi} g(\phi) e^{-i\left(q 2^{p / 2}+R\left(1-2^{-p+1}\right)\right) \phi} W_{2^{p / 2}}(\theta-\phi) d \phi,
$$

then

$$
g(\theta)=e^{i R\left(1-2^{-p+1}\right) \theta} \sum_{q=0}^{2^{-3 p / 2} R} e^{i q 2^{p / 2} \theta} g_{q}(\theta) .
$$

We observe that if $\Phi_{p}$ is a function on $\mathbb{S}^{1}$ satisfying

$$
\widehat{\Phi}_{p}(j)=\left\{\begin{array}{cc}
1 & |j| \leq 2^{p / 2+1} \\
0 & |j| \geq 2^{p / 2+2}
\end{array}\right.
$$

then, $g_{q}=\Phi_{p} * g_{q}$ for each $q$. We note that $\Phi_{p}$ may be chosen such that for each $N \in \mathbb{N}$, the $k$ th derivative of $\Phi_{p}$, denoted by $\Phi_{p}^{(k)}$, satisfies

$$
\left|\Phi_{p}^{(k)}(\phi)\right| \lesssim \frac{2^{(k+1) p / 2}}{\left(1+2^{p / 2}|\phi|\right)^{N}}
$$

for all $\phi \in[-\pi, \pi]$. (For our purposes (19) with $N=3$ and $0 \leq k \leq 2$ will be sufficient.) With this notation,

$$
\begin{aligned}
\widehat{g d \sigma}\left(R e^{i \phi}\right) & =\sum_{q=0}^{2^{-3 p / 2} R} \int_{-\pi}^{\pi} e^{i R\left(1-2^{-p+1}+q 2^{p / 2} R^{-1}\right) \theta} e^{i R \cos (\theta-\phi)} \Phi_{p} * g_{q}(\theta) d \theta \\
& =e^{i R\left(1-2^{-p+1}\right) \phi} \sum_{q=0}^{2^{-3 p / 2} R} e^{i q 2^{p / 2} \phi} g_{q} * \Psi_{p, q}(\phi),
\end{aligned}
$$


where

$$
\Psi_{p, q}(\phi)=\int_{-\pi}^{\pi} e^{i R\left[\left(1-2^{-p+1}+q 2^{p / 2} R^{-1}\right) \theta+\cos \theta\right]} \Phi_{p}(\phi-\theta) d \theta .
$$

Consequently,

$$
\begin{aligned}
\int_{-\pi}^{\pi}\left|\widehat{g d \sigma}\left(R e^{i \phi}\right)\right|^{2} \psi_{2}(\phi) d \theta & \\
= & \int_{-\pi}^{\pi} \sum_{q, q^{\prime}} e^{i\left(q-q^{\prime}\right) 2^{p / 2} \phi} g_{q} * \Psi_{p, q}(\phi) \overline{g_{q^{\prime}} * \Psi_{p, q^{\prime}}(\phi)} \psi_{2}(\phi) d \phi \\
= & \sum_{q, q^{\prime}} \int_{-\pi}^{\pi} \int_{-\pi}^{\pi} g_{q}(u) \overline{g_{q^{\prime}}(v)} \\
& \quad \times\left(\int_{-\pi}^{\pi} \Psi_{p, q}(\phi-u) \overline{\Psi_{p, q^{\prime}}(\phi-v)} \psi_{2}(\phi) e^{i\left(q-q^{\prime}\right) 2^{p / 2} \phi} d \phi\right) d u d v .
\end{aligned}
$$

Now, $\widehat{\Psi}_{p, q}(k), \widehat{\Psi}_{p, q^{\prime}}(k), \widehat{\psi}_{2}(k)=0$ for $|k| \geq 4.2^{p / 2}$, and so

$$
\int_{-\pi}^{\pi} \Psi_{p, q}(\phi-u) \overline{\Psi_{p, q^{\prime}}(\phi-v)} \psi_{2}(\phi) e^{i\left(q-q^{\prime}\right) 2^{p / 2} \phi} d \phi=0
$$

whenever $\left|q-q^{\prime}\right|>12$. Since $\left|g_{q}(u) g_{q^{\prime}}(v)\right| \leq \frac{1}{2}\left(\left|g_{q}(u)\right|^{2}+\left|g_{q^{\prime}}(v)\right|^{2}\right)$, by symmetry it suffices to control

$$
\begin{aligned}
& \sum_{\left|q-q^{\prime}\right| \leq 12} \int_{-\pi}^{\pi}\left|g_{q}(u)\right|^{2}\left(\int_{-\pi}^{\pi} \int_{-\pi}^{\pi}\left|\Psi_{p, q}(\phi-u)\right|\left|\Psi_{p, q^{\prime}}(\phi-v)\right| \psi_{2}(\phi) d \phi d v\right) d u \\
& =\sum_{\left|q-q^{\prime}\right| \leq 12} \int_{-\pi}^{\pi}\left|g_{q}(u)\right|^{2}\left(\int_{-\pi}^{\pi}\left|\Psi_{p, q}(\phi-u)\right| \psi_{2}(\phi) d \phi\right)\left(\int_{-\pi}^{\pi}\left|\Psi_{p, q^{\prime}}(v)\right| d v\right) d u
\end{aligned}
$$

The following lemma, which is easily proved by induction, will facilitate our estimates for $\left|\Psi_{p, q}\right|$.

Lemma 8 Suppose $\lambda \in \mathbb{R}, h(\theta)=\lambda \theta+\cos \theta$, and $q_{0} \in C^{\infty}\left(\mathbb{S}^{1}\right)$. If $\left\{q_{k}\right\}$ is given recursively by

$$
q_{k+1}(\theta)=\frac{d}{d \theta}\left(\frac{1}{h^{\prime}(\theta)} q_{k}(\theta)\right),
$$

then each $q_{k}$ may be expressed as a linear combination of terms of the form

$$
\frac{\cos ^{m} \theta \sin ^{n} \theta q_{0}^{(r)}(\theta)}{h^{\prime}(\theta)^{\ell}}
$$

where $r+2 \ell-m \leq 3 k$ and $2 \ell-m \geq 2 k$.

\section{Lemma 9}

$$
\left|\Psi_{p, q}(\phi)\right| \lesssim \frac{2^{p / 4}}{R^{1 / 2}} H_{p}(\phi-\pi / 2)
$$


uniformly in $q$, where $H_{p}$ satisfies

$$
H_{p}(\phi) \lesssim \frac{2^{p / 2}}{\left(1+2^{p / 2}|\phi|\right)^{N}}
$$

for each $N \in \mathbb{N}$.

(We point out that we will only require the case $N=3$ of this lemma.)

Proof. Let $\lambda_{p q}=1-2^{-p+1}+q 2^{p / 2} R^{-1}$, and let $\left\{\eta_{n}\right\}_{n \in \mathbb{N}}$ be a smooth partition of unity on $\mathbb{S}^{1}$ such that $\operatorname{supp}\left(\eta_{n}\right) \subset\left\{\theta:|\theta| \sim 2^{-n}\right\}$. For uniformity purposes let us suppose that $\left\{\eta_{n}\right\}$ is constructed in the standard way from a fixed smooth function and taking differences. We now let

$$
\eta_{p, j}= \begin{cases}\eta_{p / 2-j}, & \text { if } 0<j \leq p / 2 \\ \sum_{n \geq p / 2} \eta_{n}, & \text { if } j=0 .\end{cases}
$$

Thus for each $p,\left\{\eta_{p, j}\right\}_{0 \leq j \leq p / 2}$ forms a partition of unity on $\mathbb{S}^{1}$.

For each $j$ we let

$$
\Psi_{p, q, j}(\phi)=\int_{-\pi}^{\pi} e^{i R\left[\lambda_{p q} \theta+\cos \theta\right]} \Phi_{p}(\phi-\theta) \eta_{p, j}(\phi-\theta) d \theta,
$$

and write

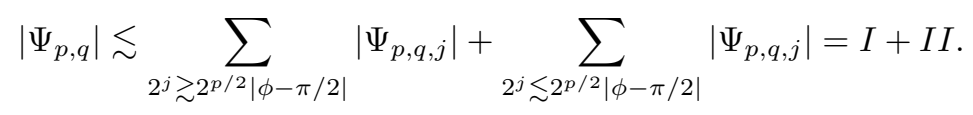

In order to bound $I$ by the required quantity it is enough to prove the uniform estimate

$$
\left|\Psi_{p, q, j}\right| \lesssim 2^{-N j} \frac{2^{3 p / 4}}{R^{1 / 2}}
$$

for each $N \in \mathbb{N}$. To this end we let $h(\theta)=\lambda_{p q} \theta+\cos \theta$, and integrate by parts as follows.

$$
\begin{aligned}
\Psi_{p, q, j}(\phi) & =\int_{-\pi}^{\pi} \frac{d}{d \theta}\left(\int_{-\pi}^{\theta} e^{i R h(t)} d t\right) \Phi_{p}(\phi-\theta) \eta_{p, j}(\phi-\theta) d \theta \\
& =J_{R \lambda_{p q}}(R) \Phi_{p}(\phi) \eta_{p, j}(\phi) \\
& -\int_{-\pi}^{\pi} J_{R \lambda_{p q}}^{\theta}(R) \frac{d}{d \theta}\left(\Phi_{p}(\phi-\theta) \eta_{p, j}(\phi-\theta)\right) d \theta,
\end{aligned}
$$

where

$$
J_{k}^{\theta}(R):=\int_{-\pi}^{\theta} e^{i(k t+R \cos t)} d t .
$$

Estimate (23) now follows from the decay estimates (19) and the uniform (in $q$ ) estimate

$$
\sup _{\theta \in[-\pi, \pi]}\left|J_{R \lambda_{p q}}^{\theta}(R)\right| \lesssim \frac{2^{p / 4}}{R^{1 / 2}}
$$


This last Bessel function estimate is a direct consequence of Lemma 5 .

We now turn to $I I$. Fix a $j$ and suppose that $2^{p / 2-j}|\phi-\pi / 2| \geq c$, for some appropriately large absolute constant $c$. For all $\theta$ in the support of the integrand defining $\Psi_{p, q, j}, 2^{p / 2-j}|\phi-\theta| \lesssim 1$, and so $|\theta-\pi / 2| \sim|\phi-\pi / 2|$. It is now easy to show that for all such $\theta, h^{\prime}(\theta) \gtrsim|\phi-\pi / 2|^{2}$, and $|\cos \theta| \lesssim|\phi-\pi / 2|$.

Now, if we define the differential operator $D$ by

$$
D=\frac{1}{i R h^{\prime}(\theta)} \frac{d}{d \theta}
$$

then for each $k \in \mathbb{N}$,

$$
\left|\Psi_{p, q, j}\right| \lesssim \int_{-\pi}^{\pi}\left|\left({ }^{t} D\right)^{k}\left\{\Phi_{p}(\phi-\theta) \eta_{p, j}(\phi-\theta)\right\}\right| d \theta .
$$

By Lemma 8, (25) is bounded by a sum of terms (the number of terms depending only on $k$ ) of the form

$$
\frac{1}{R^{k}} \int_{-\pi}^{\pi}\left|\frac{\cos ^{m} \theta \sin ^{n} \theta\left\{\Phi_{p}(\phi-\theta) \eta_{p, j}(\phi-\theta)\right\}^{(r)}}{h^{\prime}(\theta)^{\ell}}\right| d \theta
$$

where $r+2 \ell-m \leq 3 k$ and $2 \ell-m \geq 2 k$. Using the decay estimate (19), and the fact that $2^{p} \leq R^{2 / 3}$, we see that for each $k, N \in \mathbb{N}$,

$$
\begin{aligned}
(26) & \lesssim \frac{1}{R^{k}} \frac{1}{|\phi-\pi / 2|^{2 \ell-m}}\left(2^{p / 2}\right)^{r+1} 2^{-N j} 2^{-p / 2+j} \\
& \leq\left(\frac{2^{p}}{R^{2 / 3}}\right)^{3(k-1 / 2) / 2} 2^{-(N-1) j} \frac{2^{p / 4}}{R^{1 / 2}} \frac{2^{p / 2}}{\left(2^{p / 2}|\phi-\pi / 2|\right)^{2 \ell-m}} \\
& \leq 2^{-(N-1) j} \frac{2^{p / 4}}{R^{1 / 2}} \frac{2^{p / 2}}{\left(2^{p / 2}|\phi-\pi / 2|\right)^{2 k}} .
\end{aligned}
$$

The desired bound for $I I$ now follows.

The "rotation" through $\pi / 2$ described in the above lemma has recently been a feature in other works of the authors; see Section 6 of [BBC] and Section 3 of $[\mathrm{BV}]$.

Returning now to the proof of Proposition 6 , it follows immediately from Lemma 9 that

$$
\int_{-\pi}^{\pi}\left|\Psi_{p, q^{\prime}}(v)\right| d v \lesssim \frac{2^{p / 4}}{R^{1 / 2}}
$$

uniformly in $q^{\prime}$. Hence if we set $\psi_{3}=H_{p} * \psi_{2}$, then by (21) and (22),

$$
\int_{-\pi}^{\pi}\left|\widehat{g d \sigma}\left(R e^{i \phi}\right)\right|^{2} \psi_{2}(\phi) d \theta \lesssim \frac{2^{p / 2}}{R} \int_{-\pi}^{\pi} \sum_{q}\left|g_{q}(u)\right|^{2} \psi_{3}(u-\pi / 2) d u .
$$

In order to proceed with $(27)$ we need the following Littlewood-Paley type lemma. 
Lemma 10 If $L>1$ and $f_{k}=f *\left(e^{i k L \cdot} W_{L}\right)$, then, for any non-negative $w$ on $\mathbb{S}^{1}$

$$
\int_{-\pi}^{\pi} \sum\left|f_{k}\right|^{2} w \lesssim \int_{-\pi}^{\pi}|f|^{2}\left|W_{L}\right| * w .
$$

Proof.

We begin by observing that

$$
f_{k}(\theta)=e^{i k L \theta} \int_{-\pi}^{\pi} f(\phi) e^{-i k L \phi} W_{L}(\theta-\phi) d \phi,
$$

and so $\left|f_{k}(\theta)\right|$ is the absolute value of the $k L$ 'th Fourier coefficient of $f W_{L}(\theta-\cdot)$.

Since the operator

$$
g \mapsto \sum_{k} \widehat{g}(k L) e^{i k L}
$$

is convolution on the circle with the distribution

$$
\begin{aligned}
& \frac{1}{L} \sum_{j=0}^{L-1} \delta\left(\cdot-\frac{2 \pi j}{L}\right) \\
& \sum_{k}\left|f_{k}(\theta)\right|^{2}=\sum_{k}\left|\left(f W_{L}(\theta-\cdot)\right)(k L)\right|^{2} \\
&=\int_{-\pi}^{\pi}\left|\frac{1}{L} \sum_{j=0}^{L-1} f\left(\phi-\frac{2 \pi j}{L}\right) W_{L}\left(\theta-\phi+\frac{2 \pi j}{L}\right)\right|^{2} d \phi \\
& \leq \int_{-\pi}^{\pi} \frac{1}{L^{2}} \sum_{j=0}^{L-1}\left|W_{L}\left(\theta-\phi+\frac{2 \pi j}{L}\right)\right| \sum_{l=0}^{L-1}\left|W_{L}\left(\theta-\phi+\frac{2 \pi l}{L}\right)\right|\left|f\left(\phi-\frac{2 \pi l}{L}\right)\right|^{2} d \phi \\
&=\frac{1}{L^{2}} \sum_{j=0}^{L-1} \sum_{l=0}^{L-1} \int_{-\pi}^{\pi}\left|W_{L}\left(\theta-\phi+\frac{2 \pi j}{L}\right)\right|\left|W_{L}\left(\theta-\phi+\frac{2 \pi l}{L}\right)\right|\left|f\left(\phi-\frac{2 \pi l}{L}\right)\right|^{2} d \phi \\
&=\frac{1}{L^{2}} \sum_{j=0}^{L-1} \sum_{l=0}^{L-1} \int_{-\pi}^{\pi}\left|W_{L}\left(\theta-\phi+\frac{2 \pi(j-l)}{L}\right)\right|\left|W_{L}(\theta-\phi)\right||f(\phi)|^{2} d \phi \\
&=\int_{-\pi}^{\pi}\left(\left.\frac{1}{L^{2}} \sum_{j=0}^{L-1} \sum_{l=0}^{L-1}\left|W_{L}\left(\theta-\phi+\frac{2 \pi(j-l)}{L}\right)\right||| W_{L}(\theta-\phi)|| f(\phi)\right|^{2} d \phi .\right.
\end{aligned}
$$

However, by (17),

$$
\frac{1}{L^{2}} \sum_{j=0}^{L-1} \sum_{l=0}^{L-1}\left|W_{L}\left(\theta-\phi+\frac{2 \pi(j-l)}{L}\right)\right|
$$

is uniformly bounded, and so

$$
\sum_{k}\left|f_{k}(\theta)\right|^{2} \lesssim\left|W_{L}\right| *|f|^{2}(\theta)
$$


uniformly in $\theta$. Consequently,

$$
\int_{-\pi}^{\pi} \sum_{k}\left|f_{k}\right|^{2} w \lesssim \int_{-\pi}^{\pi}|f|^{2}\left|W_{L}\right| * w,
$$

as required.

By Lemma 10 applied to (27) we now have that

$$
\int_{-\pi}^{\pi}\left|\widehat{g d \sigma}\left(R e^{i \phi}\right)\right|^{2} \psi_{2}(\phi) d \theta \lesssim \frac{1}{R} \int_{-\pi}^{\pi}|g(\theta)|^{2} 2^{p / 2} \psi_{4}(\theta-\pi / 2) d \theta,
$$

where $\psi_{4}=\left|W_{2^{p / 2}}\right| * \psi_{3}$. It remains to show (c.f. (16)) that $2^{p / 2} \psi_{4}(\cdot-\pi / 2)$ is pointwise dominated by $\mathcal{M}_{p}(\mu)$. Recalling that $\psi_{3}=H_{p} * \Theta_{p} * \psi_{1}$, we first seek to understand the averaging operator $P_{p} * \mu(\theta-\pi / 2)$ in geometrical terms.

Lemma 11 Let $\mathcal{C}_{\lambda}$ denote the arc on $\mathbb{S}^{1}$ centred at $-\pi / 2$ of length $2 \lambda 2^{p} / R$. Let $1 \leq 2^{p} \leq R^{2 / 3}$ and $1 \leq \lambda \leq R / 2^{p}$. Then $\mu\left(\mathcal{C}_{\lambda}\right) \leq 4 \lambda^{2} \sup _{T} \mu(T)$, where the sup is taken over all rectangles $T$ of sides $2^{p / 2} / R \times 2^{p} / R$ with long side parallel to the $x$-axis.

Proof. $\mathcal{C}_{\lambda}$ is contained in the axis parallel rectangle centred at $(0,-1)$ of horizontal side $2 \lambda 2^{p} / R$ and vertical side $2\left(\lambda 2^{p} / R\right)^{2}$. Therefore $\mathcal{C}_{\lambda}$ is contained in at most

$$
\left(2 \lambda 2^{p} / R\right)^{2}\left(2^{p / 2} / R\right)^{-1} \leq 4 \lambda^{2}
$$

of the permitted rectangles $T=T\left(2^{p / 2} / R, 2^{p} / R\right)$.

\section{Corollary 12}

for all $\theta \in[-\pi, \pi]$.

$$
2^{p / 2} P_{p} * \mu(\theta-\pi / 2) \lesssim \mathcal{M}_{p} \mu(\theta)
$$

Proof. By rotational symmetry we may assume that $\theta=0$. By (14), the kernel $P_{p}$ is dominated by

$$
\sum_{1 \leq 2^{k} \leq R / 2^{p}} \frac{1}{2^{k N}} \frac{\chi_{\left\{|\cdot| \leqslant 2^{k} 2^{p} / R\right\}}}{2^{k} 2^{p} / R}
$$

(for example with $N=2$ ). Thus, by Lemma 11,

$$
\begin{aligned}
P_{p} * \mu(-\pi / 2) & \lesssim \frac{R}{2^{p}} \sum_{1 \leq 2^{k} \leq R / 2^{p}} \frac{\mu\left(\mathcal{C}_{2^{k}}\right)}{2^{k(N+1)}} \\
& \lesssim \frac{R}{2^{p}}\left(\sum_{1 \leq 2^{k} \leq R / 2^{p}} \frac{2^{2 k}}{2^{k(N+1)}}\right) \sup _{T} \mu(T),
\end{aligned}
$$

where the supremum is taken over all rectangles $T=T\left(2^{p / 2} / R, 2^{p} / R\right)$ with long side parallel to the $x$-axis. Hence

$$
2^{p / 2} P_{p} * \mu(-\pi / 2) \lesssim \sup _{T \|(1,0)} \frac{\mu\left(T\left(2^{p / 2} / R, 2^{p} / R\right)\right)}{2^{p / 2} / R}=\mathcal{M}_{p} \mu(0) .
$$


Lemma 13 If $\nu$ is a measure on the unit ball $\mathbb{B}$, then

$$
\mathcal{M}_{p}(\nu)(\theta-\phi) \lesssim\left(1+2^{p / 2}|\sin \phi|\right) \mathcal{M}_{p}(\nu)(\theta) .
$$

Proof. Take a typical rectangle $T=T\left(2^{p / 2} / R, 2^{p} / R\right)$ parallel to $\theta-\phi$, and cover it with rectangles $S=S\left(2^{p / 2} / R, 2^{p} / R\right)$ which are parallel to $\theta$.

It follows directly from Corollary 12 and Lemma 13 that $2^{p / 2} \psi_{1}(\cdot-\pi / 2) \lesssim$ $\mathcal{M}_{p}(\mu)$. Next we observe that if $\chi_{p}$ is a bump function at scale $2^{-p / 2}$ satisfying

$$
\left|\chi_{p}(\phi)\right| \lesssim \frac{2^{p / 2}}{\left(1+2^{p / 2}|\phi|\right)^{\ell}}
$$

for some $\ell>2$, and $\nu$ is any measure on $\mathbb{B}$, then by Lemma 13

$$
\begin{aligned}
\chi_{p} * \mathcal{M}_{p}(\nu)(\theta) & \lesssim \mathcal{M}_{p}(\nu)(\theta) \int\left(1+2^{p / 2}|\phi|\right)\left|\chi_{p}(\phi)\right| d \phi \\
& \lesssim \mathcal{M}_{p}(\nu)(\theta) \int \frac{2^{p / 2}}{\left(1+2^{p / 2}|\phi|\right)^{\ell-1}} d \phi \lesssim \mathcal{M}_{p}(\nu)(\theta) .
\end{aligned}
$$

Since $\psi_{2}, \psi_{3}$ and $\psi_{4}$ are obtained by successive convolutions with such functions at scale $2^{-p / 2}$, we may conclude that $2^{p / 2} \psi_{4}(\cdot-\pi / 2) \lesssim \mathcal{M}_{p}(\mu)$. This completes the proof of part 1 of Proposition 6.

We now turn to part 2, which is considerably easier. We have already (indirectly) dealt with the case where $g$ has frequencies supported in $\mathcal{C}_{0}$ - we simply reproduce the argument from part 1 with $p=1$. If $g$ has frequencies supported in $\mathcal{C}_{\infty}$, then we argue in the following standard way. Let $\Phi$ be a member of the Schwartz class $\mathcal{S}\left(\mathbb{R}^{2}\right)$ such that $\widehat{\Phi}$ is identically 1 on the ball of radius 2 in $\mathbb{R}^{2}$. Since $|\widehat{g d \sigma}(R \cdot)|^{2}=|\widehat{g d \sigma}|^{2} * \Phi_{1 / R}$, where $\Phi_{1 / R}=R^{2} \Phi(R \cdot)$,

$$
\int_{\mathbb{S}^{1}}|\widehat{g d \sigma}(R \omega)|^{2} d \mu(\omega)=\int_{\mathbb{R}^{2}}|\widehat{g d \sigma}(R x)|^{2} \Phi_{1 / R} * d \mu(x) d x .
$$

Here $*$ denotes convolution on $\mathbb{R}^{2}$. Using polar coordinates and the rapid decay of $\Phi$ it therefore suffices to prove the required inequality, (12), where $\mu$ is essentially constant at scale $1 / R$. In this case,

$$
\begin{aligned}
\int_{\mathbb{S}^{1}}|\widehat{g d \sigma}(R x)|^{2} d \mu(x) & \leq\|\mu\|_{\infty}\|\widehat{g d \sigma}(R \cdot)\|_{2}^{2} \leq\|\mu\|_{\infty} \sup _{|j| \geq 3 R / 2}\left|J_{j}(R)\right|^{2}\|g\|_{2}^{2} \\
& \lesssim \frac{1}{R} \int_{\mathbb{S}^{1}}|g(\omega)|^{2} \mathcal{M}_{0}(\mu)(\omega) d \sigma(\omega) .
\end{aligned}
$$

Here we have used the fact that $\sup _{|j| \geq 3 R / 2}\left|J_{j}(R)\right| \lesssim R^{-1 / 2}$ and that $\mathcal{M}_{0}(\mu)$ is constant and of the order of $\|\mu\|_{\infty}$. 


\section{Conclusion of the Proof of Theorem 2}

The following Littlewood-Paley type proposition will allow us to deduce Theorem 2 from Proposition 6.

Proposition 14 For each $k \in \mathbb{N}$, let $Q_{k} \in C^{1}\left(\mathbb{S}^{1}\right)$ be such that $\operatorname{supp}\left(\widehat{Q}_{k}\right) \subset$ $\left\{j \in \mathbb{Z}:|j| \sim 2^{k}\right\}$. Suppose further that for each $N \in \mathbb{N}$ there is a constant $C_{N} \geq 0$ such that

$$
\left|Q_{k}(\theta)\right|+2^{-k}\left|Q_{k}^{\prime}(\theta)\right| \leq C_{N} \frac{2^{k}}{\left(1+2^{k}|\theta|\right)^{N}}
$$

for all $k$.

Then

$$
\int_{\mathbb{S}^{1}} \sum_{k}\left|f * Q_{k}\right|^{2} w \lesssim \int_{\mathbb{S}^{1}}|f|^{2} M w .
$$

Proof. The proof we give is based on an argument from Chang, Wilson and Wolff $[\mathrm{CWW}]$.

For each $j \in \mathbb{Z}$, let

$$
E_{j}=\left\{x \in \mathbb{S}^{1}: M w(x)>2^{j}\right\}
$$

and for each $k \in \mathbb{Z}$, let

$$
A_{j k}=\left\{x \in \mathbb{S}^{1}: B\left(x, 2^{-k}\right) \subset E_{j}\right\} .
$$

We now choose another (similar) bump function $\widetilde{Q}_{k}$ at scale $2^{-k}$ such that $\widetilde{Q}_{k} * Q_{k}=Q_{k}$ for every $k$. Now, by Jensen's inequality,

$$
\begin{gathered}
\int_{\mathbb{S}^{1}} \sum_{k}\left|f_{k}\right|^{2} w \lesssim \int_{\mathbb{S}^{1}} \sum_{k}\left|Q_{k} * f\right|^{2}\left|\widetilde{Q}_{k}\right| * w \\
=\sum_{j} \sum_{k} \int_{A_{j k} \backslash A_{j+1, k}}\left|Q_{k} * f\right|^{2}\left|\widetilde{Q}_{k}\right| * w,
\end{gathered}
$$

and since $\left|\widetilde{Q}_{k}\right| * w(x) \lesssim 2^{j}$ whenever $x \in A_{j k} \backslash A_{j+1, k},(30)$ is bounded by

$$
\sum_{j} 2^{j} \sum_{k} \int_{A_{j k}}\left|Q_{k} * f\right|^{2} .
$$

Now let $\left\{P_{n}\right\}_{n \in \mathbb{N}}$ be a smooth partition of unity on $\mathbb{S}^{1}$ such that $\operatorname{supp}\left(P_{n}\right) \subset$ $\left\{\theta:|\theta| \sim 2^{-n}\right\}$. For uniformity purposes let us suppose that $\left\{P_{n}\right\}$ is constructed in the standard way by scaling a certain fixed smooth function and taking differences. For $k, \ell \in \mathbb{N}$ with $0 \leq \ell \leq k$ we now define

$$
P_{k, \ell}= \begin{cases}P_{k-\ell}, & \text { if } 0<\ell \leq k \\ \sum_{n \geq k} P_{n}, & \text { if } \ell=0\end{cases}
$$


Thus for each $k,\left\{P_{k, \ell}\right\}_{0 \leq \ell \leq k}$ forms a partition of unity on $\mathbb{S}^{1}$.

By elementary geometric considerations,

$$
\left(Q_{k} P_{k, \ell}\right) * f(x)=\left(Q_{k} P_{k, \ell}\right) *\left(\chi_{E_{j-\ell}} f\right)(x)
$$

for all $x \in A_{j k}$, and so by Plancherel's Theorem,

$$
\begin{aligned}
\left(\sum_{j} 2^{j}\right. & \left.\sum_{k} \int_{A_{j k}}\left|Q_{k} * f\right|^{2}\right)^{1 / 2} \\
& =\left(\sum_{j} 2^{j} \sum_{k} \int_{A_{j k}}\left|\sum_{\ell}\left(Q_{k} P_{k, \ell}\right) *\left(f \chi_{E_{j-\ell}}\right)\right|^{2}\right)^{1 / 2} \\
& \leq\left(\sum_{j} 2^{j} \sum_{k} \sum_{m}\left|\sum_{\ell} \widehat{Q_{k} P_{k, \ell}}(m) \widehat{f \chi_{E_{j-\ell}}}(m)\right|^{2}\right)^{1 / 2} \\
& \leq \sum_{\ell}\left(\sum_{j} 2^{j} \sum_{k} \sum_{m}\left|\widehat{Q_{k} P_{k, \ell}}(m) \widehat{f \chi_{E_{j-\ell}}}(m)\right|^{2}\right)^{1 / 2} \\
& =\sum_{\ell}\left(\sum_{j} 2^{j} \sum_{m}\left(\sum_{k}\left|\widehat{Q_{k} P_{k, \ell}}(m)\right|^{2}\right)\left|\widehat{f \chi_{E_{j-\ell}}}(m)\right|^{2}\right)^{1 / 2} .
\end{aligned}
$$

Fix $m \in \mathbb{Z}$. If $m 2^{\ell-k} \leq 1$, then for each $N \in \mathbb{N}$,

$$
\left|\widehat{Q_{k} P_{k, \ell}}(m)\right|=\left|\int_{-\pi}^{\pi} Q_{k} P_{k, \ell}(\theta)\left[e^{i m \theta}-1\right] d \theta\right| \leq 2^{\ell-k} m 2^{-\ell(N-1)},
$$

and so,

$$
\sum_{k: m 2^{\ell-k} \leq 1}\left|\widehat{Q_{k} P_{k, \ell}}(m)\right|^{2} \lesssim 2^{-2 \ell(N-1)} .
$$

On the other hand, if $m 2^{\ell-k}>1$, then integrating by parts once we obtain

$$
\left|\widehat{Q_{k} P_{k, \ell}}(m)\right| \leq \frac{1}{m} \int_{-\pi}^{\pi}\left|\left(Q_{k} P_{k, \ell}\right)^{\prime}(\theta)\right| d \theta \leq \frac{1}{m} 2^{k} 2^{-\ell(N-1)}=\frac{2^{-\ell(N-2)}}{m 2^{\ell-k}} .
$$

Hence by (33) and (34),

$$
\sum_{k}\left|\widehat{Q_{k} P_{k, \ell}}(m)\right|^{2} \lesssim 2^{-2 \ell(N-2)}
$$

for each $N \in \mathbb{N}$, and so by Plancherel's Theorem again, (32) is (up to a multiplicative constant) bounded by

$$
\sum_{\ell \in \mathbb{N}} 2^{-\ell(N-2)}\left(\sum_{j} 2^{j} \sum_{m}\left|\widehat{f \chi_{E_{j-\ell}}}(m)\right|^{2}\right)^{1 / 2}
$$




$$
=\sum_{\ell \in \mathbb{N}} 2^{-\ell(N-5 / 2)}\left(\sum_{j} 2^{j-\ell} \int|f|^{2} \chi_{E_{j-\ell}}\right)^{1 / 2} \lesssim\left(\int|f|^{2} M w\right)^{1 / 2} .
$$

We note that we only appeal to the case $N=3$ of hypothesis (29).

We now pass to the situation for a general $g$ with unrestricted frequency support. Let $A_{p}^{+}, A_{p}^{-}, B_{p}^{+}, B_{p}^{-}, C_{0}$ and $C_{\infty}$ be appropriate (smoothed-out) Littlewood-Paley convolution operators associated with the intervals $\mathcal{A}_{p},-\mathcal{A}_{p}$, $\mathcal{B}_{p},-\mathcal{B}_{p}, \mathcal{C}_{0}$ and $\mathcal{C}_{\infty}$ respectively. That is

$$
\begin{gathered}
g=\sum_{p} A_{p}^{+} g+\sum_{p} A_{p}^{-} g+\sum_{p} B_{p}^{+} g+\sum_{p} B_{p}^{-} g+C_{0} g+C_{\infty} g \\
=g_{A^{+}}+g_{A^{-}}+g_{B^{+}}+g_{B^{-}}+g_{0}+g_{\infty} .
\end{gathered}
$$

Now, $\widehat{g_{A^{+}} d \sigma}=\sum_{p} \widehat{A_{p}^{+} g d \sigma}$ and so

$$
\left|\widehat{g_{A^{+}} d \sigma}\right|^{2} \lesssim \log R \sum_{p}\left|\widehat{A_{p}^{+} g d \sigma}\right|^{2}
$$

Hence, by Propositions 6 and 14,

$$
\begin{aligned}
\int_{\mathbb{S}^{1}}\left|\widehat{g_{A^{+}} d \sigma}(R x)\right|^{2} d \mu(x) & \lesssim \frac{\log R}{R} \int \sum_{p}\left|A_{p}^{+} g\right|^{2} \mathcal{M}_{p}(\mu) d \sigma \\
& \lesssim \frac{\log R}{R} \int|g|^{2} M \mathcal{M}^{R}(\mu) d \sigma .
\end{aligned}
$$

A similar treatment of $g_{A^{-}}, g_{B^{+}}, g_{B^{-}}, g_{0}$ and $g_{\infty}$ finishes the proof of (9).

We now turn to $(10)$. Since $\overrightarrow{g d \sigma}(R x)$, with $x=(\cos \theta, \sin \theta)$ is precisely convolution on $\mathbb{S}^{1}$ of $g$ with $e^{i R \cos }$, we may apply classical Littlewood-Paley theory (of a similar nature to Proposition 14 - see $[\mathrm{W}]$ and $[\mathrm{CP}]$ ), along with Proposition 6, to obtain

$$
\begin{aligned}
\int_{\mathbb{S}^{1}}\left|\widehat{g_{A^{+}} d \sigma}(R x)\right|^{2} d \mu(x) & \lesssim \int_{\mathbb{S}^{1}} \sum_{p}\left|\widehat{A_{p}^{+} g d \sigma}(R x)\right|^{2} M^{2}(\mu) d \sigma(x) \\
& \lesssim \frac{1}{R} \int_{\mathbb{S}^{1}} \sum_{p}\left|A_{p}^{+} g(\omega)\right|^{2} \mathcal{M}_{p} M^{2}(\mu)(\omega) d \omega,
\end{aligned}
$$

where $M^{2}$ denotes the two-fold composition $M \circ M$. Again by Proposition 14 we may conclude (10), completing the proof of Theorem 2 .

\section{Proof of Corollary 3}

Although Corollary 3 is a simple consequence of Theorem 2, a direct proof may be given which, in particular, does not require the second frequency decomposition (18). The main ingredients of this we now sketch. If $g$ has Fourier 
frequencies supported in $\mathcal{A}_{p}$, then by (13), Plancherel's Theorem on $\mathbb{S}^{1}$, and Lemma 5,

$$
\begin{aligned}
\int_{\mathbb{S}^{1}}|\widehat{g d \sigma}(R x)|^{2} d \mu(x) & \leq\left\|P_{p} * \mu\right\|_{\infty}\|\widehat{g d \sigma}(R \cdot)\|_{2}^{2} \\
& \leq\left\|P_{p} * \mu\right\|_{\infty} \sup _{j \in \mathcal{A}_{p}}\left|J_{j}(R)\right|^{2}\|g\|_{2}^{2} \\
& \lesssim \frac{1}{R} 2^{p / 2}\left\|P_{p} * \mu\right\|_{\infty}\|g\|_{2}^{2},
\end{aligned}
$$

which by Corollary 12, is bounded by an absolute constant multiple of

$$
\frac{1}{R}\|g\|_{2}^{2} \sup _{R^{-1} \leq \alpha \leq R^{-2 / 3}} \frac{\mu\left(T\left(\alpha, \alpha^{2} R\right)\right)}{\alpha},
$$

uniformly in $p$. Treating $g$ with frequencies in the remaining terms of our initial frequency decomposition (i.e. $-\mathcal{A}_{p}, \mathcal{B}_{p},-\mathcal{B}_{p}, \mathcal{C}_{0}$ and $\mathcal{C}_{\infty}$ ) in a similar way, completes the proof of Corollary 3. It is not clear at this stage whether the logarithmic term may be removed here.

\section{Proof of Corollary 4}

By Hölder's inequality and duality, it suffices to prove that

$$
\left\|\mathcal{M}^{R}(\psi d \sigma)\right\|_{L^{3}\left(\mathbb{S}^{1}\right)} \lesssim R^{1 / 3}\|\psi\|_{L^{3}\left(\mathbb{S}^{1}\right)}
$$

for all $g \in L^{3}\left(\mathbb{S}^{1}\right)$ and $R \geq 1$.

For each $j \in \mathbb{N}$ with $1 \leq 2^{j} \leq R^{1 / 3}$, let

$$
M_{j}^{R} w(\theta)=\sup _{2^{2 j} \leq 2^{p} \leq R^{2 / 3}} \sup _{|\theta-\eta| \lesssim 2^{j-p / 2}} \frac{1}{2^{p / 2} R^{-1}} \int_{|\eta-\phi| \lesssim 2^{-j+p} R^{-1}} w(\phi \pm \pi / 2) d \phi,
$$

where $w(\phi \pm \pi / 2)=w(\phi+\pi / 2)+w(\phi-\pi / 2)$. Since $\mathcal{M}^{R}(\psi d \sigma) \lesssim \sup _{j} M_{j}^{R} \psi$, we seek estimates for $\left\|M_{j}^{R} \psi\right\|_{3}$ which have an appropriate geometric decay in $2^{j}$. (For convenience we work with functions on $\mathbb{R}$ rather than $\mathbb{S}^{1}$.) To this end we let

$$
\Gamma_{R}^{j}(t)=\left\{(x, y) \in \mathbb{R} \times \mathbb{R}^{+}: 0<y<2^{-j} R^{-1 / 3},|x-t|^{2} y<2^{j} R^{-1}\right\},
$$

and

$$
\psi_{\beta, j}^{*}(t)=\sup _{(x, y) \in \Gamma_{R}^{j}(t)} y^{\beta}\left|\Phi_{y} * \psi(x)\right|,
$$

where $\Phi$ is a compactly supported smooth bump function at scale $1, \Phi_{y}=$ $\frac{1}{y} \Phi(\dot{\dot{y}})$, and $\beta \geq 0$. Since

$$
M_{j}^{R} \psi(\theta) \lesssim R^{1 / 2} 2^{-j / 2}\left(\psi_{\frac{1}{2}, j}^{*}(\theta+\pi / 2)+\psi_{\frac{1}{2}, j}^{*}(\theta-\pi / 2)\right),
$$


it will suffice to show that

$$
\left\|\psi_{\frac{1}{2}, j}^{*}\right\|_{3} \lesssim 2^{j / 6} R^{-1 / 6}\|\psi\|_{3} .
$$

(Maximal operators of the form (38), corresponding to certain tangential approach regions, have already been studied by Nagel and Stein [NS]. However, the regions $\Gamma_{R}^{j}$ that we consider fail to satisfy the conditions that they impose.) By Stein's analytic interpolation theorem (see [S1]), in order to obtain (39) it will be enough to prove the estimates

$$
\left\|\psi_{\frac{3}{2}, j}^{*}\right\|_{L^{1}} \lesssim 2^{j / 2} R^{-1 / 2}\|\psi\|_{H^{1}}
$$

and

$$
\left\|\psi_{0, j}^{*}\right\|_{\infty} \lesssim\|\psi\|_{\infty}
$$

Inequality (41) is immediate, whereas inequality (40) can be verified quickly by testing on atoms. More specifically, if $a$ is an $H^{1}$-atom with corresponding support interval $I$ (by translation invariance we may suppose that $I$ is centred at the origin), then by standard arguments we obtain the pointwise bound

$$
\left|\Phi_{y} * a(x)\right| \lesssim \begin{cases}1 /|I|, & \text { if } y \lesssim|I|, \quad|x| \lesssim|I| \\ |I| / y^{2}, & \text { if } y \gtrsim|I|, \quad y \gtrsim|x| \\ 0, & \text { otherwise. }\end{cases}
$$

The subsequent pointwise bounds for the corresponding $a_{\frac{3}{2}, j}^{*}$ quickly lead to the required estimate

$$
\left\|a_{\frac{3}{2}, j}^{*}\right\|_{L^{1}} \lesssim 2^{j / 2} R^{-1 / 2}
$$

completing the proof.

Although Corollary 4 is optimal within the family of all such $L^{p}-L^{q}$ inequalities, it is considerably less subtle than the $L^{2}$ weighted inequality from which it came (inequality (10) of Theorem 2). It is likely that weighted inequalities of a similar nature can be obtained for certain more general oscillatory integral operators, such as those treated by Greenleaf and Seeger in [GS]. This would require a deeper geometric understanding of the action of such an operator, which would then be encoded in an associated "characteristic" maximal function.

\section{References}

[BBC] J. A. Barceló, J. M. Bennett and A. Carbery, A bilinear extension inequality in two dimensions, Journal of Functional Analysis, Vol. 201 (2003), 1, 57-77.

[BRV] J. A. Barceló, A. Ruiz and L. Vega, Weighted estimates for the Helmholtz equation and consequences, Journal of Functional Analysis, Vol. 150 (1997), 2, 356-382. 
[BV] J. M. Bennett and A. Vargas, Randomised circular means of Fourier transforms of measures, Proc. Amer. Math. Soc. 131 (2003), 117-127.

[CP] D. Cruz-Uribe and C. Pérez, Two weight extrapolation via the maximal operator, Journal of Functional Analysis, Vol. 174 (2000), 1-17.

[CRS] A. Carbery, E. Romera and F. Soria, Radial weights and mixed norm inequalities for the disc multiplier, Journal of Functional Analysis, Vol. 109, (1992), 1, 52-75.

[CS1] A. Carbery and F. Soria, Pointwise Fourier inversion and localisation in $\mathbb{R}^{n}$, Journal of Fourier Analysis and Applications 3, special issue (1997), $847-858$.

[CS2] A. Carbery and F. Soria, Sets of divergence for the localisation principle for Fourier integrals, C. R. Acad. Sci. Paris. Sér. I Math. 325, no. 12 (1997), 1283-1286.

[CSV] A. Carbery, F. Soria and A. Vargas, Localisation and weighted inequalities for spherical Fourier means, preprint.

[CWW] S. Y. A. Chang, J. M. Wilson, T. H. Wolff, Some weighted norm inequalities concerning the Schrödinger operators, Comment. Math. Helvetici, 60 (1985), 217-246.

[GS] A. Greenleaf and A. Seeger, On oscillatory integral operators with folding canonical relations, Studia Math. 132 (1999), 2, 125-139.

[NS] A. Nagel and E. M. Stein, On certain maximal functions and approach regions, Adv. in Math., 54 (1984), 1, 83-106.

[R] E. Romera, Tesis Doctoral, Universidad Autónoma de Madrid, 1991.

[S] E. M. Stein, Some problems in harmonic analysis, Proc. Sympos. Pure Math., Williamstown, Mass., (1978), Part 1, 3-20.

[S1] E. M. Stein, Harmonic Analysis, Princeton University Press, 1993.

[W] J. M. Wilson, Weighted norm inequalities for the continuous square functions, Trans. Amer. Math. Soc. 314 (1989), 661-692. 